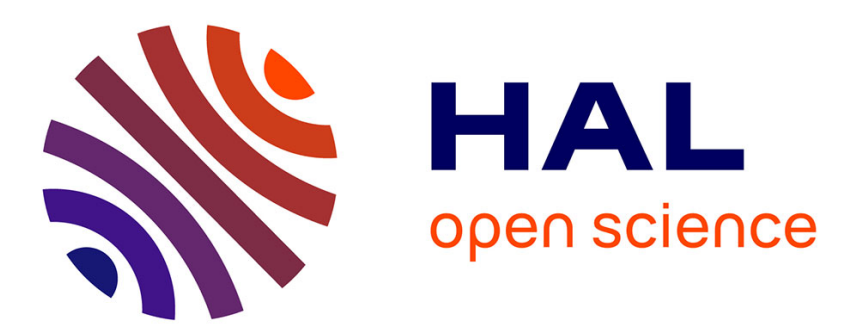

\title{
A propos de l'évolution de la contamination du lait par des résidus de pesticides organochlorés entre 1970 et
} 1976

\author{
H. Mahieu, F. M. Luquet, L. Mouillet
}

\section{- To cite this version:}

H. Mahieu, F. M. Luquet, L. Mouillet. A propos de l'évolution de la contamination du lait par des résidus de pesticides organochlorés entre 1970 et 1976. Le Lait, 1977, 57 (568), pp.547-560. hal00928770

\section{HAL Id: hal-00928770 \\ https://hal.science/hal-00928770}

Submitted on 1 Jan 1977

HAL is a multi-disciplinary open access archive for the deposit and dissemination of scientific research documents, whether they are published or not. The documents may come from teaching and research institutions in France or abroad, or from public or private research centers.
L'archive ouverte pluridisciplinaire HAL, est destinée au dépôt et à la diffusion de documents scientifiques de niveau recherche, publiés ou non, émanant des établissements d'enseignement et de recherche français ou étrangers, des laboratoires publics ou privés. 


\title{
A propos de l'évolution de la contamination du lait par des résidus de pesticides organochlorés entre 1970 et 1976
}

\author{
par
}

H. MAHIEU*, F. M. LUQUET** et L. MOUILLET ${ }^{* *}$

\section{TABLE DES MATIERES}

INTRODUCTION.

EcHANTILLONS ET MÉTHODES.

RÉSULTATS ET COMMENTAIRES.

CONCLUSION.

AnNexes :

Les causes de la contamination.

La contamination des aliments.

La législation internationale.

La législation française.

Mesures à prendre pour éviter la contamination.

Conseils à tout utilisateur de produits de traitement.

BIBLIOGRAPHIE.

**

Travail effectué dans le cadre des études et enquêtes financées par l'I.T.E.B.

Période 1970-1974 : laits de producteurs. Période 1974-1976 : laits de grand mélange.

* I.T.E.B., 149, rue de Bercy - 75579 Paris cedex 12.

** Labcodral, 105, rue de l'Université - 59509 Douai. 


\section{INTRODUCTION}

Le mot " pesticide " (1) est le mot le plus couramment employé, en France tout au moins, pour désigner les produits antiparasitaires, naturels ou de synthèse, utilisés :

- en agriculture ;

- lors du stockage de denrées alimentaires destinées aux humains ou aux animaux ;

- en médecine vétérinaire ;

- en médecine et hygiène humaines (assainissement, lutte contre les maladies épidémiques...) ;

- en industrie (imprégnation des bois, protection de la laine...).

Ces parasites peuvent aussi bien être des rongeurs que des insectes, des mauvaises herbes, des champignons, des micro-organismes, etc.

La nature chimique des " pesticides " est très diverse :

- produits naturels, tels la roténone, le pyrèthre ;

- substances chimiques simples : sulfate de cuivre, acide sulfurique... ;

- produits de synthèse contenant du chlore (les organochlorés), du phosphore (les organophosphorés), des métaux ( $\mathrm{Sn}, \mathrm{Hg}, \mathrm{Ca} . .$. ) ;

- produits dérivés des phénols et des crésols ;

- produits nitrés, soufrés ;

- produits dérivés de l'acide carbamique ;

- produits dérivés des huiles de goudron ou de benzène ;

- fumigants...

Les "pesticides " ont un rôle très précis, leur emploi est conditionné par la nature des végétaux à traiter, des parasites à combattre...

Les plus utilisés en France en 1975 ont été :

- herbicides 44,5 p. 100

- fongicides 24,3 p. 100 du marché des produits

- insecticides 22,7 p. 100

- divers $\quad 8,5$ p. 100 antiparasitaires

Pour le propos qui nous concerne ici, il convient de distinguer (bien que la distinction ne soit pas aussi nette) :

- les pesticides biodégradables, c'est-à-dire se détruisant d'euxmêmes assez rapidement au niveau des sols, de l'atmosphère et des eaux ou dans les tissus végétaux et animaux : c'est le cas par exemple des produits à base d'éléments organophosphorés ;

- les pesticides à dégradation très lente donc à forte rémanence : c'est le cas des produits à base d'éléments organochlorés. Ils présentent une forte rémanence pouvant aller jusqu'à plusieurs années. Ces

(1) Dérivé du mot anglais «pest »: fléau. 
substances, presque exclusivement apportées par l'emploi des insecticides, sont très liposolubles; elles peuvent s'accumuler et se rencontrer dans les matières grasses. Ceci se produit à tous les niveaux : sols, eaux, végétaux, aliments, chez les animaux et chez l'homme lui-même.

La rémanence des pesticides organochlorés ainsi que leur faculté à se fixer dans les graisses a pour conséquence la contamination du beurre et des fromages par les résidus des produits de traitement. Ces résidus sont-ils dangereux pour la santé humaine ? Certainement pas aux doses infinitésimales rencontrées dans les produits laitiers. La consommation courante des boissons alcoolisées n'a pas de peine à causer plus de dégâts sur le corps humain. Il n'empêche qu'une certaine psychose de pollution amène le consommateur à s'interroger sur la qualité des produits dès lors qu'ils contiennent des résidus de produits étrangers à la nature même de l'aliment. La profession laitière se devait donc de considérer ce problème.

Lors d'une enquête en 1955, la Food And Drug Administration des Etats-Unis décela dans 62 p. 100 des échantillons de lait qu'elle avait à examiner une contamination par des résidus de " pesticides " organochlorés.

La F.D.A. commença alors une recherche sur les principales causes de pollution des produits laitiers par de tels résidus, recherche qui aboutit en 1962 au rassemblement d'un grand nombre de données techniques.

En Europe, ce sont les danois qui abordèrent les premiers ce problème en 1961, au Laboratoire Municipal de Aarhus. Le sondage s'étant révélé positif, ils étendirent l'enquête à tout le pays en 1964.

En France, les produits laitiers firent l'objet de sondages dès 1965 par les équipes des professeurs Mestres, Thieulin, Viel et Casalis. Par la suite, des études systématiques sur la pollution des produits laitiers furent entreprises par des laboratoires officiels spécialisés :

- Laboratoire de Phytopharmacie de l'I.N.R.A. ;

- Chaire d'Industrie Laitière de l'E.N.S.I.A.A. à Douai ;

- Laboratoire des Services Vétérinaires ;

et par des laboratoires d'usines laitières, telles que :

- Fromagerie Bel ;

- Roquefort ;

- Genvrain ;

- Union Laitière Normande, etc.

Plusieurs pays ont imposé de ce fait des teneurs maximales en résidus, teneurs généralement établies en tenant compte des seuils de tolérance conseillés par l'Office Mondial de la Santé et la F.A.O. (voit tab. 1). L'application de ces normes n'est pas sans poser de 
H.C.H. $\left\{\begin{array}{l}\text { Total } \\ \alpha+\beta \\ \text { Lindane }\end{array}\right.$

Heptachlore et son époxyde

Aldrine + Dieldrine

Aldrine + Dieldrine + Endrine

Endrine

Chlordane

D.D.T. + D.D.D. + D.D.E.

H.C.B.

Toxaphène

P.C.B.
Tolérances administratives $* * *$

\begin{tabular}{|c|c|c|c|c|c|c|}
\hline D.J.A.** & $\begin{array}{l}\text { Recomman- } \\
\text { dations***}\end{array}$ & U.S.A. & Canada & Suisse & R.F.A. & Pays-Bas \\
\hline & & 300 & 300 & 200 & 200 & 300 \\
\hline 10 & 100 & & 100 & 200 & 200 & 200 \\
\hline 0,5 & 150 & 300 & 125 & 125 & 150 & 125 \\
\hline 0,1 & 150 & 300 & 100 & 125 & 150 & 125 \\
\hline 0,2 & 20 & & & & 20 & 20 \\
\hline 1 & 50 & & 100 & & 50 & 50 \\
\hline 5 & 1250 & 1250 & 1000 & 1250 & 1000 & 1250 \\
\hline \multirow[t]{3}{*}{0,6} & 500 & 300 & 100 & 300 & 500 & 300 \\
\hline & & 100 & 100 & & 400 & \\
\hline & & 2500 & & 2500 & 2500 & \\
\hline
\end{tabular}

* Etat de la réglementation au 31 janvier 1977.

** D.J.A. : Doses journalières acceptables maximales exprimées en $\mu \mathrm{g} / \mathrm{kg}$ de poids corporel.

*** Quantités de résidus exprimées en $\mu \mathrm{g} / \mathrm{kg}$ de M.G. pure (microgrammes par kilogramme). 
problèmes pour le commerce extérieur français, dans lequel les produits laitiers représentent une part importante.

Difficulté d'autant plus grave que la profession laitière subit un préjudice pour un phénomène dont elle n'est que très partiellement responsable.

Afin de déterminer les diverses sources de contamination des produits laitiers par les "pesticides " et de mieux délimiter les diverses responsabilités la profession laitière et l'Institut Technique de l'Elevage Bovin (I.T.E.B.) ont décidé en 1970, à la suite d'une préenquête menée par l'U.P.C.I.L., la réalisation d'enquêtes approfondies. Ces enquêtes devaient tenir compte des résultats obtenus par les laboratoires aussi bien officiels que privés qui ont recherché la teneur en résidus de pesticides organochlorés des produits finis : beurre et fromages.

PREMIÈre PARTIE

\section{ECHANTILLONS ET METHODES}

\section{LES ECHANTILLONNAGES}

L'Institut Technique de l'Elevage Bovin a organisé depuis 1970 un réseau de correspondants (cent-cinquante producteurs et soixantedix usines) afin de pouvoir mener à bien sur le terrain les études et enquêtes concernant les différents problèmes qui lui sont posés (1).

C'est à ces réseaux que l'I.T.E.B. s'est adressé pour suivre l'évolution de la contamination du lait par des résidus de pesticides organochlorés :

- au niveau de la production, en 1970-1974;

- sur des laits de grand mélange, en 1974-1976.

\section{Echantillonnage au niveau de la production}

Deux enquêtes successives ont été réalisées. La première s'est déroulée de 1970 à 1972. Elle avait pour but de définir les processus les plus fréquents par lesquels les pesticides organochlorés peuvent contaminer le lait à des taux excédants les limites généralement admises (cf. annexes).

La deuxième enquête s'est déroulée de 1972 à 1974. Elle a permis, sur un échantillon plus important de préciser certains des processus

(1) Le Lait, nov.-déc. 1976, n 559-560, p. 660 à 671. 
et de suivre l'évolution de la contamination sur une plus longue période.

Les deux enquêtes ont été organisées au niveau de l'exploitation agricole ; les exploitations choisies répondant à deux critères :

- le lait livré à la laiterie est contaminé par des résidus de pesticides organochlorés ;

- l'exploitation possède suffisamment d'enregistrements sur son fonctionnement pour qu'il soit possible de pousser toutes les investigations nécessaires à la recherche des causes de contamination.

Les producteurs visités ont été choisis par les laiteries qui ont bien voulu participer à ces deux premières enquêtes. Des études importantes ayant, durant cette période, été montées en Normandie et en Bretagne, ces deux régions ont été exclues.

Avant ou lors du premier prélèvement, le responsable de l'étude est passé chez chaque producteur afin de procéder à une enquête concernant principalement :

- les caractéristiques de l'exploitation ;

- les caractéristiques de l'élevage ;

- l'alimentation des vaches laitières ;

- les amendements utilisés.

Ces fiches «producteurs » ont servi, par la suite, à vérifier les relations entre la pollution et l'alimentation, en liaison avec le laboratoire phyto-pharmacie du C.N.R.A. à Versailles (MM. Viel et Hascoët) (1).

\section{TABLEAU 2}

Echantillonnage laits de producteurs (enquêtes 1970-1972 et 1972-1974)

\begin{tabular}{l|c|c}
\hline & $1970-1972$ & $1972-1974$ \\
\cline { 2 - 3 } Centre-Est & 19 & 32 \\
Est & 12 & 13 \\
Nord-Est & 15 & 24 \\
Ouest & 28 & 27 \\
Sud-Ouest & 12 & 24 \\
Massif Central & - & 11 \\
\hline France & 86 & 131 \\
\hline
\end{tabular}

(1) Le Lait, nov.-déc. 1976, nº 559-560, p. 660 à 671. 
L'étude a été réalisée dans six régions, chez une centaine de producteurs choisis dans les tournées, d'une vingtaine de laiteries (tab. 2).

Les fiches producteurs étaient mises à jour lors de chaque prélèvement :

- hiver 1970-1971 préenquête 1970-1972

- février 1971 juin 1971 septembre 1971 mars 1972

- juin 1972

- octobre 1972 mars 1973 octobre 1973 mars 1974

TABLEAU 3. - Echantillonnage 1974-1976

\begin{tabular}{|c|c|c|c|}
\hline Régions & $\begin{array}{c}\text { Nombre } \\
\text { de } \\
\text { départements }\end{array}$ & $\begin{array}{l}\text { Nombre } \\
\text { d'usines }\end{array}$ & $\begin{array}{l}\text { Représentativité } \\
\text { de l'échantillon } \\
\text { en p. } 1000 \\
\text { de la collecte }\end{array}$ \\
\hline Centre-Est & 10 & 11 & 6,5 \\
\hline Est & 13 & 10 & 3,1 \\
\hline Nord-Est & 10 & 9 & 2,8 \\
\hline Normandie & 5 & 7 & 4,0 \\
\hline Bretagne & 4 & 5 & 4,0 \\
\hline Ouest & 11 & 10 & 5,0 \\
\hline Sud-Ouest & 13 & 7 & 4,5 \\
\hline $\begin{array}{l}\text { Centre } \\
\text { Massif Central }\end{array}$ & 12 & 6 & 1,3 \\
\hline Sud & 12 & 0 & 0 \\
\hline France & 90 & 65 & 4,0 \\
\hline
\end{tabular}

enquête 1970-1972 et préenquête 1972-1974 (nouveaux producteurs)

enquête 1972-1974 
TABLEAU 4. - Enquête 1974-1976 (répartition des usines et litrages correspondants)

\begin{tabular}{|c|c|c|c|c|c|c|c|c|c|c|c|c|c|c|c|c|}
\hline & \multicolumn{6}{|c|}{1974} & \multicolumn{6}{|c|}{1975} & \multicolumn{4}{|c|}{1976} \\
\hline & \multicolumn{2}{|c|}{ février } & \multicolumn{2}{|c|}{ juin } & \multicolumn{2}{|c|}{ octobre } & \multicolumn{2}{|c|}{ février } & \multicolumn{2}{|c|}{ juin } & \multicolumn{2}{|c|}{ octobre } & \multicolumn{2}{|c|}{ mars } & \multicolumn{2}{|c|}{ octobre } \\
\hline & (1) & $(2)$ & (1) & $(2)$ & (1) & (2) & (1) & $(2)$ & (1) & $(2)$ & (1) & (2) & (1) & (2) & (1) & (2) \\
\hline Centre-Est & 10 & 2500 & 11 & 3800 & 7 & 1650 & 10 & 2400 & 5 & 1800 & 6 & 2300 & 7 & 7000 & 2 & 1250 \\
\hline Est & 7 & 4665 & 8 & 6613 & 8 & 4090 & 6 & 4425 & 6 & 5594 & 10 & 9852 & 8 & 7953 & 9 & 7292 \\
\hline Nord-Est & 3 & 4722 & 5 & 6420 & 5 & 3563 & 3 & 4817 & - & - & 9 & 6846 & 5 & 6004 & 10 & 14446 \\
\hline Normandie & 7 & 5100 & 6 & 5639 & 7 & 4074 & 5 & 4134 & 6 & 5301 & 5 & 3546 & 4 & 3851 & 9 & 5842 \\
\hline Bretagne & 2 & 2637 & 5 & 9439 & 4 & 6452 & 4 & 5779 & 2 & 3700 & 5 & 15500 & 5 & 13069 & 5 & 12130 \\
\hline Ouest & 10 & 12862 & 10 & 18450 & 10 & 12877 & 9 & 14153 & 8 & 16959 & 7 & 12392 & 6 & 13953 & 11 & 13673 \\
\hline Sud-Ouest & 4 & 4144 & 5 & 5532 & 4 & 4179 & 2 & 2710 & 4 & 5703 & 7 & 6584 & 4 & 4380 & 5 & 5519 \\
\hline Centre - Massif Central & 4 & 1600 & 4 & 1450 & 3 & 1100 & 2 & 1600 & 2 & 1450 & 6 & 4910 & 7 & 4342 & 8 & 3719 \\
\hline \multirow[t]{2}{*}{ France } & 47 & 38230 & 54 & 57343 & 48 & 37985 & 41 & 40018 & 33 & 40507 & 56 & $61 \subseteq 30$ & 46 & 60552 & 59 & 63871 \\
\hline & \multicolumn{6}{|c|}{149 usines pour $133558 \mathrm{hl}$} & \multicolumn{6}{|c|}{130 usines pour $142455 \mathrm{hl}$} & \multicolumn{4}{|c|}{105 usines pour $124423 \mathrm{~h}$} \\
\hline (1) Nombre d'usines & \multicolumn{6}{|c|}{ (2) Litrage collecté (hl) } & & & & & & & & & & \\
\hline
\end{tabular}


A chaque passage était prélevé dans un flacon en verre bouché émeri, un échantillon de $500 \mathrm{ml}$ de lait. Les échantillons, additionnés de bichromate de potassium (1 g/l) étaient envoyés pour analyse au laboratoire d'industrie laitière de Douai.

Simultanément en février 1971, septembre 1971, mars 1972 et mars 1973 un échantillon de chaque aliment distribué aux animaux a été prélevé et envoyé, pour analyse, au laboratoire de phytopharmacie du C.N.R.A. à Versailles.

\section{Echantillonnage au niveau de l'usine : 1974-1976 (laits de grand mélange)}

En 1974, il a été demandé aux usines ayant participé depuis l'année 1970 aux enquêtes sur les résidus de pesticides organochlorés, de collaborer à ces nouvelles études. Soixante-cinq usines ou centres de ramassage ont répondu positivement.

La représentativité de l'échantillon en p. 1000 de la collecte, fut variable selon les zones, les prélèvements et les analyses. La somme des laits ramassés par les différentes usines participant à l'enquête représente environ 4 p. 1000 d'une collecte nationale journalière moyenne (tab. 3).

Dans chaque usine, chaque prélèvement était effectué sur un lait de grand mélange, c'est-à-dire correspondant à l'ensemble des laits arrivant normalement à la laiterie considérée.

Huit prélèvements ont été effectués (tab. 4) :

février, juin et octobre 1974

février, juin et octobre 1975

mars et octobre $\quad 1976$

A chaque prélèvement, deux flacons en matière plastique de $250 \mathrm{ml}$ étaient remplis. Le conservateur utilisé était le bichromate de potassium ( $1 \mathrm{~g} / \mathrm{l})$. Les échantillons sitôt constitués étaient envoyés, pour analyse, au Laboratoire d'Industrie Laitière de Douai (devenu le Labcodral). Sur la fiche accompagnatrice étaient transcrits :

- le volume de lait correspondant à l'échantillon ;

- un code propre à l'échantillon, au prélèvement et à la laiterie.

$\mathrm{Au}$ cas où des analyses simultanées étaient effectuées par les laiteries, il était demandé que les résultats soient transmis au responsable de l'étude.

\section{LES METHODES DE DOSAGE}

La première méthode de dosage des résidus de pesticides organochlorés décrite ci-après est une variante de celle proposée par Langlois, Stemp et Liska. C'est une méthode rapide de contrôle qui 


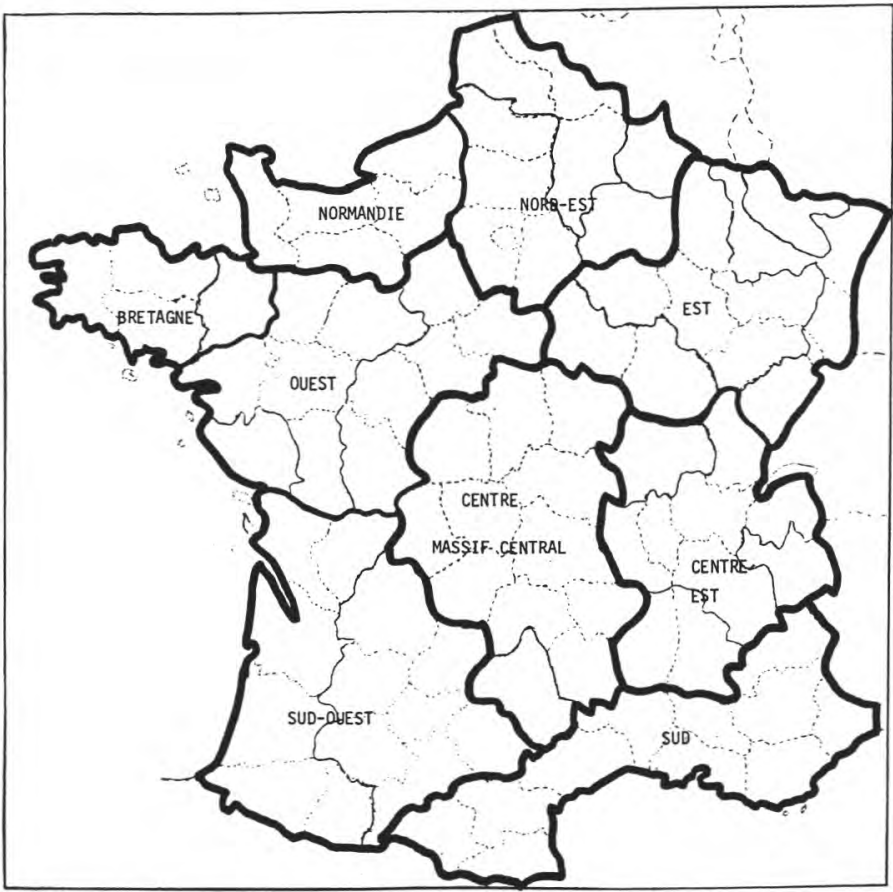

fig. 1

Répartition de l'échantillon

a été appliquée systématiquement aux échantillons des enquêtes 1970-1972 et 1972-1974 prélevés chez les producteurs.

La deuxième méthode de dosage est celle testée lors des chaînes analytiques internationales et décrite par la Fédération Internationale de Laiterie et la Food And Drug Administration. Cette deuxième méthode a été appliquée systématiquement aux échantillons des enquêtes 1974-1976 prélevés sur des laits de grand mélange ; et a été utilisée en tant que contrôle pour les résultats donnés par la méthode de Goursaud-Luquet-Casalis.

1. Méthode rapide de contrôle de la teneur en résidus de pesticides organochlorés dans le lait (3) (méthode Goursaud - Luquet Casalis)

(3) Principe de la méthode et équipement. Le Lait, nov.-déc. 1968, $\mathrm{n}^{\circ}$ 479-480, p. 645 à 654 . 


\section{Dosage des insecticides organochlorés dans le lait (méthode} F.I.L.-F.D.A. utilisée au Labcodral)

\section{A. PRINCIPE}

La phase grasse qui contient les insecticides est extraite suivant une technique différente selon qu'il s'agit de lait, de beurre ou de fromage.

Pour le lait, cette extraction est faite d'abord à l'acétone, puis à l'éther de pétrole.

La phase grasse subit ensuite une purification préalable par partage acétonitrile-hexane, complétée par une purification sur colonne de florisil.

Le dosage proprement dit est réalisé par chromatographie en phase gazeuse sur deux colonnes de «polarité » différente.

B. Matériel

Ampoules à décanter de $250 \mathrm{ml}, 500 \mathrm{ml}, 1000 \mathrm{ml}$, équipées de robinets plats (ou de robinets à boisseau munis de carottes en Téflon), et de bouchons rodés en verre.

Broyeur "Sorvall » à pot de $400 \mathrm{ml}$ en inox (ou matériel similaire).

Evaporateur rotatif.

Colonne à chromatographie d'une longueur au moins égale à $40 \mathrm{~cm}$ et d'un diamètre intérieur de $16 \mathrm{~mm}$ (extérieur : $20 \mathrm{~mm}$ ), rodée à la partie supérieure $(19 / 26)$, et munie de robinet plat (ou de robinet à boisseau muni de carotte en Téflon).

Ampoule cylindrique à robinet rodé (carotte verre ou Téflon), et rodage inférieur s'adaptant sur la colonne à chromatographie $(19 / 26)$.

Appareil de chromatographie en phase gazeuse (C.G.L.), muni d'un détecteur à capture d'électrons et équipé comme suit :

Colonne :

pyrex diamètre intérieur : $<4 \mathrm{~mm}$. longueur : 1,50 $\mathrm{m}$ ou 1,80 $\mathrm{m}$ (5-6 pieds).

Injection dans la colonne ou chambre d'injection en pyrex.

Phase stationnaire : 1) DOW 11 ou DC 200.

2) $\mathrm{QF}_{1}$ ou $\mathrm{OV} 210$.

Granulométrie : $\quad 100-120$ mesh.

Taux d'imprégnation : 5 p. 100. 
Support :

Varoport ou équivalent (GAS CMROM Q). gaz vecteur : azote. débit : $40 \mathrm{ml} / \mathrm{mn}$.

\section{Solvants}

1. Qualité des solvants

Ether de pétrole :

redistillé sur pyrex.

Hexane :

Acétone pure :

Acétonitrile : redistillé sur pyrex. redistillée sur pyrex. redistillé sur pyrex.

Eau distillée agitée avec de l'éther de pétrole, redistillée (eau : dix parties, hexane : une partie) ou eau naturelle ayant satisfait aux essais de pureté.

\section{Essais de pureté}

Solvants organiques. Pour tous les solvants, amener $300 \mathrm{cc}$ du solvant à sec, reprendre par $10 \mathrm{cc}$ d'hexane redistillé et passer en C.G.L. sur les deux colonnes de référence. Une injection de $5 \mu \mathrm{l}$ ne doit donner aucun pic d'une hauteur supérieure à 5 p. 100 de l'échelle $(1 \mathrm{~cm} / 20 \mathrm{~cm})$ (le pic "d'injection » mis à part).

Eau distillée. $500 \mathrm{cc}$ agités avec $20 \mathrm{cc}$ d'hexane. Sécher l'hexane amener à $10 \mathrm{cc}$ et passer en C.G.L. (volume d'injection : $5 \mu \mathrm{l}$ ).

Réactifs. Sulfate de Na anhydre pour analyse. Florisil : 60-100 mesh activé à $1200^{\circ} \mathrm{F}$.

Le florisil doit être maintenu à $130^{\circ} \mathrm{C}, 48 \mathrm{~h}$ avant son emploi. Laisser refroidir en flacon rodé. Ajouter ensuite 2 p. 100 d'eau, agiter et laisser reposer $24 \mathrm{~h}$.

Etant donné les variations existant entre les différents lots ; il est nécessaire de procéder à un double contrôle du florisil dans les conditions normales d'utilisation, avant sa mise en service.

Un des contrôles portera sur l'absence d'interférence et le second sur le taux de récupération pour les principaux insecticides organochlorés.

\section{Mélange éluant}

Ether de pétrole distillé + chlorure de méthylène $(2+1)$.

\section{Technioue de dosage}

\section{Extraction}

Dans un bécher de $600 \mathrm{ml}$; placer $75 \mathrm{cc}$ de lait, ajouter $150 \mathrm{ml}$ d'acétone distillée. Bien agiter. Laisser reposer quelques instants.

Filtrer sur un entonnoir garni de laine de verre, recueillir le filtrat dans une ampoule de $1000 \mathrm{ml}$. 
Rincer le bécher à l'acétone. Essorer le tampon de laine de verre (et le précipité) en les pressant contre les parois de l'entonnoir avec un gros agitateur de verre.

Placer le résidu et la laine de verre ainsi essorés dans le bécher précédemment rincé et extraire par agitation avec $2 \times 75 \mathrm{ml}$ d'éther de pétrole distillé (après filtration sur laine de verre), chacune des phases éthérées est réunie à la phase acétonique dans l'ampoule à décanter. Agiter.

Dans la même ampoule, ajouter $400 \mathrm{cc}$ d'eau salée à 20 p. 1000 .

Après un repos de $10 \mathrm{mn}$, recueillir la phase éthérée sur $\mathrm{SO}^{4} \mathrm{Na}^{2}$ sec. Evaporer à sec. Peser la M.G. ( $\neq 3$ g).

\section{Purification}

a) Partage acétonitrile-hexane. Réchauffer la M.G. précédemment pesée, et la dissoudre dans $27 \mathrm{cc}$ d'hexane purifié, saturé d'acétonitrile, et introduire quantitativement la solution dans une ampoule à décanter de $250 \mathrm{ml}$. Ajouter $90 \mathrm{ml}$ d'acétonitrile saturé d'hexane (qui auront servi auparavant à parfaire le rinçage du récipient ayant contenu la M.G.).

Agiter vigoureusement l'ampoule pendant $2 \mathrm{mn}$ exactement.

Laisser reposer $2 \mathrm{~h}$. Décanter $60 \mathrm{ml}$ de la phase acétonitrile, jeter l'hexane contenant la M.G. et le reste d'acétonitrile. Transvaser dans une ampoule de $500 \mathrm{ml}$, ajouter $10 \mathrm{ml}$ d'hexane et $250 \mathrm{ml}$ d'eau salée à 20 p. 1000 . Laisser reposer à nouveau. Décanter la phase hexanique. Laver la phase aqueuse par $10 \mathrm{ml}$ d'hexane purifiée. Grouper les phases hexaniques contenant les pesticides.

b) Purification sur colonne. Peser $25 \mathrm{~g}$ de florisil, l'introduire dans une colonne préalablement munie d'un tampon de verre. Coiffer la colonne d'un peu de $\mathrm{SO}_{4} \mathrm{Na}_{2}$ anhydre (sur une hauteur de $2-4 \mathrm{~cm}$ environ). Rincer par $50 \mathrm{ml}$ de mélange éluant, puis $50 \mathrm{ml}$ d'éther de pétrole purifié.

Introduire la phase éthérée contenant les pesticides dans la colonne en réglant l'écoulement à environ 1-2 gouttes par seconde. Dès que le niveau du liquide affleure la partie haute du $\mathrm{SO}_{4} \mathrm{Na}_{2}$, ajouter quelques millimètres d'éther de pétrole, affleurer à nouveau et éluer par $250 \mathrm{ml}$ du mélange.

Amener l'éluat juste à sec à l'évaporateur tournant (B.M. à température ambiante), reprendre à deux reprises par quelques millilitres d'hexane. Amener à $10 \mathrm{ml}$.

Dosage. Celui-ci est réalisé par C.G.L. dans les conditions opératoires suivantes:

— quantité injectée : $5 \mu 1$; 
- température du four de colonne : $180^{\circ} \mathrm{C}-200^{\circ} \mathrm{C}$ (maximum compatible avec une séparation correcte des isomères $\alpha, \beta, \gamma$, de l'H.C.H.) ;

- température de l'injecteur : environ $20^{\circ} \mathrm{C}$ au-dessus de celle du four de colonne.

\section{Calcul des résultats}

Les quantités de pesticide obtenues par dosage en C.G.L. seront corrigées en appliquant, pour chacun des composés, le facteur obtenu en faisant le produit du coefficient de partage acétonitrile/hexane, et du taux de récupération de la colonne de florisil.

Le taux de récupération des colonnes sera établi à partir de l'une d'entre elles par élution d'une solution de référence. Il est généralement supérieur à 90 p. 100.

L'ensemble des résultats est exprimé en microgramme par kilogramme de Matière Grasse Pure.

$$
\mu \mathrm{g} / \mathrm{kg} \text { de M.G.P. }
$$

(à suivre)

Reçu pour publication en mars 1977. 